\title{
Treatment of Intracranial Infection Caused by Methicillin-Resistant Staphylococcus epidermidis with Linezolid Following Poor Outcome of Vancomycin Therapy: A Case Report and Literature Review
}

\author{
Xinyang $\mathrm{Fu}^{\mathrm{l}, *}$ \\ Zhiqiang $\operatorname{Lin}^{1} * *$ \\ Sumei Chen ${ }^{2}$ \\ Limian Hong' \\ Xueping $\mathrm{Yu}^{2}$ \\ Shuifa $\mathrm{Wu}^{\prime}$
}

'Department of Pharmacy, Quanzhou First Hospital Affiliated to Fujian Medical University, Quanzhou, Fujian, People's Republic of China; ${ }^{2}$ Department of Infectious Disease, Quanzhou First Hospital Affiliated to Fujian Medical University, Quanzhou, Fujian, People's Republic of China

*These authors contributed equally to this work

\begin{abstract}
The pharmacokinetic/pharmacodynamic (PK/PD) parameter for evaluating the efficacy of vancomycin is now recommended to target an AUC/MIC (area under the curve, AUC; minimum inhibitory concentration, MIC) ratio of 400 to 600 , and trough concentration should not be used as a substitute. We report a case of intracranial infection caused by methicillin-resistant Staphylococcus epidermidis (MRSE), which was sensitive to vancomycin $(\mathrm{MIC}=2 \mu \mathrm{g} / \mathrm{mL})$ and linezolid $(\mathrm{MIC}=4 \mu \mathrm{g} / \mathrm{mL})$. The trough concentration of vancomycin in serum was $18.3 \mu \mathrm{g} / \mathrm{mL}$, and the vancomycin concentration in CSF was $5.0 \mu \mathrm{g} / \mathrm{mL}$, all within normal range. However, the AUC/MIC ratio was calculated to be $125 \mathrm{mg} \cdot \mathrm{h} \cdot \mathrm{L}^{-1}$, unable to reach target AUC/MIC. Vancomycin was replaced with linezolid after 36 days of treatment due to poor outcome, and the patient was eventually cured. Further, 23 cases of intracranial methicillin-resistant Staphylococcus aureus (MRSA) or methicillin-resistant coagulase-negative Staphylococcus (MRCoNS) infections were reported, of which 1 case with MRSA had a vancomycin MIC of $1 \mu \mathrm{g} / \mathrm{mL}$, while the remaining 22 cases had vancomycin MICs $>1 \mu \mathrm{g} / \mathrm{mL}$. The linezolid-containing regimen was used after drug susceptibility results or if the initial treatment failed, leading to recovery in 19 patients, microbial clearance in 3 patients, and treatment failure in 1 case. In conclusion, vancomycin dosing should be based on AUC-guided dosing and monitoring. When the vancomycin MIC of MRSA/MRCoNS is $>1 \mu \mathrm{g} / \mathrm{mL}$, the target AUC/MIC may not be achieved. In such cases, linezolid can effectively be considered as a good alternative to vancomycin.
\end{abstract}

Keywords: AUC/MIC, trough concentration, intracranial infection, vancomycin, linezolid

\section{Introduction}

In hospitalized patients, the integrity of the blood-brain barrier (BBB) can be disrupted by several invasive procedures, such as craniotomy, the placement ventricular catheters, lumbar puncture, and intrathecal infusions. In such situations, specific microorganisms can invade the central nervous system (CNS) through $\mathrm{BBB}$, leading to health care-associated meningitis or ventriculitis (HCAMV). ${ }^{1}$ The majority of HCAMV cases are caused by Gram-positive cocci, particularly coagulase-negative staphylococci. Infectious Diseases Society of America (IDSA) guidelines recommend vancomycin combined with anti-Pseudomonas $\beta$-lactam drugs as an empirical treatment for HCAMV. However, when the vancomycin MIC of MRSA or MRCoNS is greater than $1 \mu \mathrm{g} / \mathrm{mL}$, linezolid, daptomycin, or 
sulfamethoxazole should be considered instead. ${ }^{2}$ Herein, we report a patient with intracranial Staphylococcus epidermidis infection who recovered after vancomycin was replaced by linezolid due to poor outcome. To better investigate the sensitivity of vancomycin and the feasibility of linezolid-containing regimen, we further reviewed the available reported intracranial infections cases caused by MRSA or MRCoNS, and analyzed the efficacy of linezolid, which may hopefully help clinicians perform optimal antibiotic therapy for this kind of infection.

\section{Case Presentation}

A 67-year-old male patient was admitted to our hospital on March 20, 2020 with a complaint of sudden disturbance of consciousness. An emergency head CT scan showed 1. left basal ganglia haemorrhage with intraventricular extension, 2. bilateral lacunar infarctions at basal ganglia and senile brain degeneration, 3. mild bilateral inflammation of ethmoid sinuses. An emergency lung CT scan showed chronic bronchitis-emphysema, pulmonary bullae, and mild chronic inflammation of both lungs. Physical examination at admission showed a body temperature of $36.7^{\circ} \mathrm{C}$, a pulse of $100 \mathrm{bpm}$, a respiratory rate of 21 breaths per minute, and a blood pressure of $156 / 92 \mathrm{~mm} \mathrm{Hg}$. The patient was in a light coma and showed no response to instructions or questions. Rough respiratory sounds were detected in both lungs, and no moist rale was detected in either of the lower lungs. Neurological examination showed eye opening and vocalization with strong pricking, binocular gaze to the left side, and pupils equal in size with a diameter of $3 \mathrm{~mm}$, round, and reactive to light. No rigidity was detected in the neck and the patient could not cooperate in muscle strength examination. Voluntary movements of the left limbs were observed while no movement was observed in the right limbs. The patient could not cooperate during the examination of muscle tone or tendon reflexes. A positive Babinski sign was observed on the right side but negative on the left side, while a positive Kernig sign was present bilaterally.

Considering the clinical manifestations and examinations, a diagnosis of left basal ganglia cerebral hemorrhage and bilateral basal ganglia lacunar infarction were made. The symptomatic treatments were provided, including haemostasis, dehydration to decrease intracranial pressure, and blood pressure control. Bedside bilateral drill, craniotomy and ventricular drainage were performed. Bronchoscopy-guided nasotracheal intubation was performed. A repeat CT scan on March 21 showed that the right ventricular hematoma mostly resolved, and therefore the right ventricular drainage catheter was removed.

On March 23 (d3), the patient was still in a light coma, and moist rales in both lower lungs increased, along with an elevated white blood cell (WBC) count $\left(15.36 \times 10^{9} / \mathrm{L}\right)$ and an elevated C-reactive protein (CRP) level $(59.21 \mathrm{mg} /$ L). Pulmonary infection was diagnosed, and intravenous infusion of piperacillin-tazobactam $(4.5 \mathrm{~g}$ every $8 \mathrm{~h})$ was used as an anti-infection treatment. A repeat head CT scan on March 25 showed a significant decrease in the volume of intraventricular hematoma. In order to avoid infection after long-term retention, the drainage catheter of the left ventricle was removed.

On March 30 (d10), the patient developed fever and was in the twilight state of consciousness with aggravation of neck rigidity. The WBC count increased to $17.17 \times 10^{9}$ / $\mathrm{L}$, and the first routine and biochemical tests of cerebrospinal fluid (CSF) showed significant increases in cell count, proportion of multinucleated cells, protein level (Figures 1 and 2), and slight decrease in sugar level (Figure 2), leading to a diagnosis of possible intracranial bacterial infection. Piperacillin-tazobactam was discontinued, and the anti-infective therapy of intravenous infusion of vancomycin $(1 \mathrm{~g}$ every $12 \mathrm{~h})$ combined with ceftriaxone ( $2 \mathrm{~g}$ every $12 \mathrm{~h})$ was administered with lumbar cistern drainage performed concurrently.

The vancomycin trough serum concentration on April 3 was $18.3 \mu \mathrm{g} / \mathrm{mL}$. On April 8(d19), the patient still had fever with aggravated neck rigidity, accompanied by cough and sputum expectoration. Therefore, the combined anti-infective treatment was considered ineffective, and ceftriaxone was discontinued. A combination of vancomycin with continuous 2-hour intravenous infusion of meropenem ( 2 g every $8 \mathrm{~h}$ ) was administrated.

On April 12 (d23), the patient was in the twilight state of consciousness with no fever. The volume of lumbar cisternal drainage was $150 \mathrm{~mL}$ with unobstructed CSF drainage, and the drainage fluid was light yellow and turbid. On April 13 (d24), the number of CSF WBC was $2.325 \times 10^{9} / \mathrm{L}$, the concentration of protein in CSF was $760 \mathrm{mg} / \mathrm{L}$, and the concentration of sugar in CSF was $3.00 \mathrm{mmol} / \mathrm{L}$. Blood routine and CRP tests showed decreased WBC count and CRP. CSF routine and biochemical tests showed that cell count and protein level decreased significantly compared with previous results (Figures 1 and 2). The CSF biochemical tests showed that sugar level elevated to normal (Figure 2). On April 18, the results of the first two CSF cultures revealed the presence of Staphylococcus epidermidis, which was resistant to 


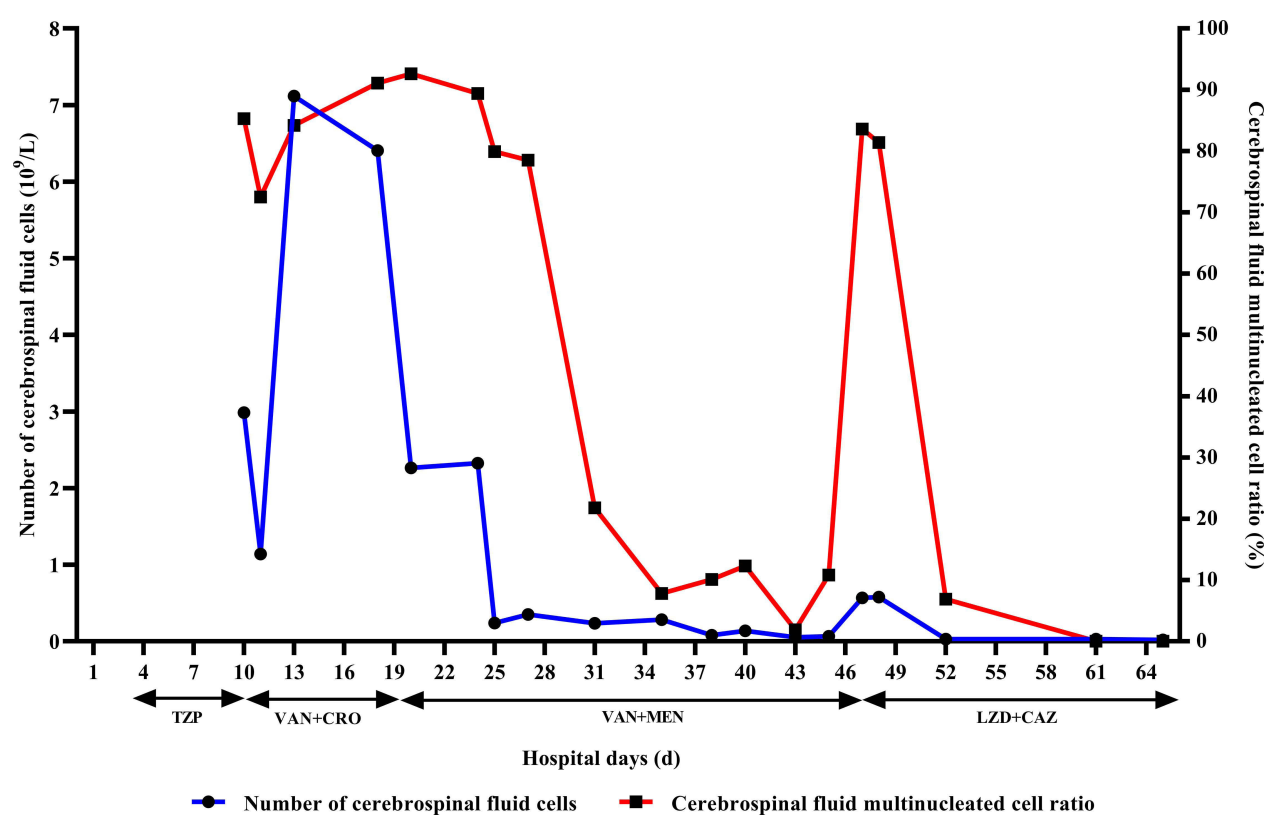

Figure I Treatment regimens and CSF cell counts.

Abbreviations: TZP, piperacillin-tazobactam; VAN, vancomycin; CRO, ceftriaxone; MEM, meropenem; LZD, linezolid (discontinued from May I0 to May II); CAZ, ceftazidime.

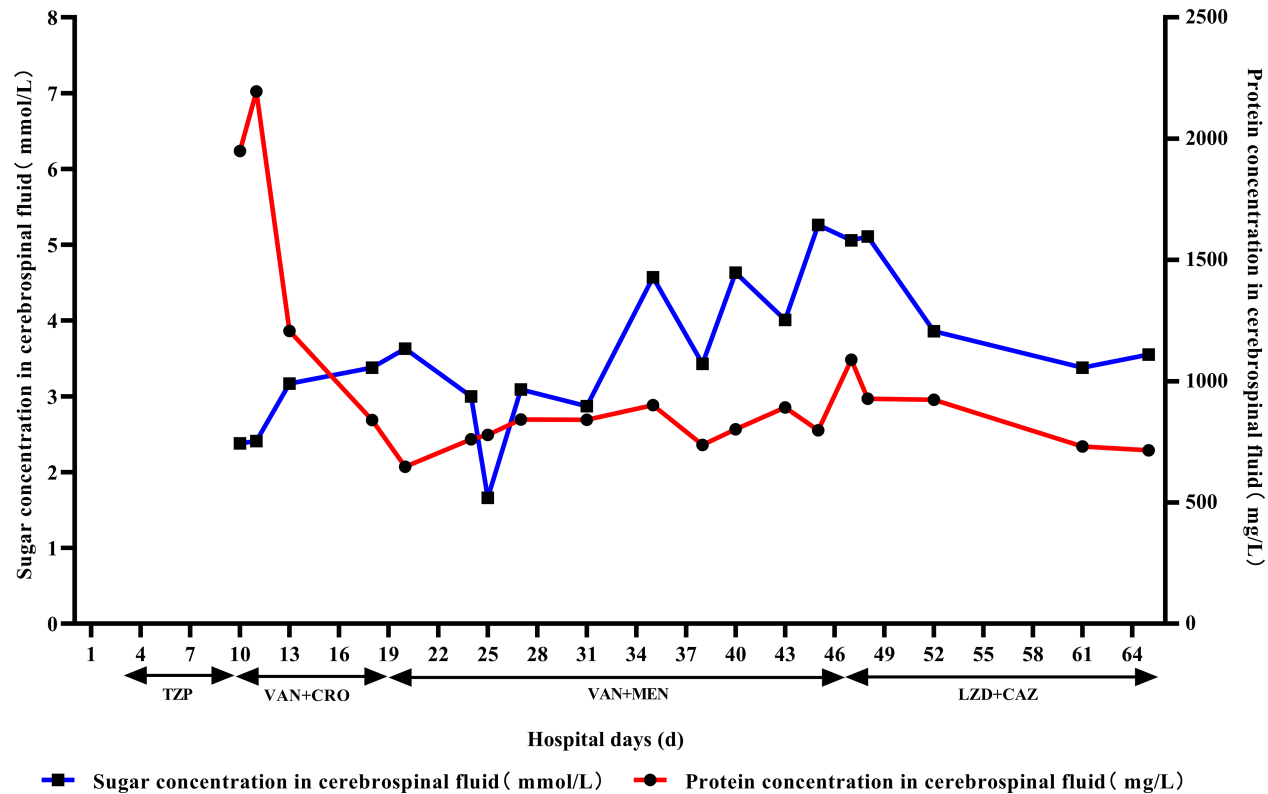

Figure 2 Treatment regimens and concentration of protein and sugar in CSF.

Abbreviations: TZP, piperacillin-tazobactam; VAN, vancomycin; CRO, ceftriaxone; MEM, meropenem; LZD, linezolid (discontinued from May 10 to May II); CAZ, ceftazidime.

oxacillin and sensitive to vancomycin ( $\mathrm{MIC}=2 \mu \mathrm{g} / \mathrm{mL}$ ) and linezolid (MIC $=4 \mu \mathrm{g} / \mathrm{mL}$ ). Both the MIC values of vancomycin and linezolid were determined by broth microdilution (BMD). The current anti-infective therapy was deemed effective, and the treatment of vancomycin combined with meropenem continued.
On April 27 (d38), patient's neck rigidity improved, the number of CSF WBC was $0.079 \times 10^{9} / \mathrm{L}$, the concentration of protein in CSF was $737 \mathrm{mg} / \mathrm{L}$, and the concentration of sugar in CSF was $3.43 \mathrm{mmol} / \mathrm{L}$. CSF routine and biochemical tests showed that cell count, proportion of multinucleated cells and protein level further decreased (Figures 1 
and 2). The lumbar cisternal drainage catheter was removed. On April 29, the CSF metagenomic sequencing revealed Staphylococcus epidermidis (read count 1541), and the treatment of vancomycin combined with meropenem continued.

On May 6 (d47), the patient developed fever again, with a maximum body temperature of $38.4^{\circ} \mathrm{C}$. The number of platelet count was $203 \times 10^{9} / \mathrm{L}$. The number of CSF WBC was $0.569 \times 10^{9} / \mathrm{L}$, the concentration of protein in CSF was $1089 \mathrm{mg} / \mathrm{L}$, and the concentration of sugar in CSF was $5.06 \mathrm{mmol} / \mathrm{L}$. CSF examination showed turbid CSF with elevated WBC count (Figure 1), increased proportion of multinucleated cells, and elevated protein level compared with previous tests (Figures 1 and 2), leading to the conclusion that the infection aggravated again. The CSF concentration of vancomycin was $5.0 \mu \mathrm{g} / \mathrm{mL}$. The anti-infective regimen was adjusted to $0.6 \mathrm{~g}$ linezolid every $12 \mathrm{~h}$ combined with $2 \mathrm{~g}$ ceftazidime every $8 \mathrm{~h}$.

On May 10, the patient's liver function test showed 528 $\mathrm{U} / \mathrm{L}$ alanine transaminase (ALT) and $323 \mathrm{U} / \mathrm{L}$ aspartate transaminase (AST). Considering possible linezolidinduced liver injury, linezolid was discontinued. On May 11 (d52), the patient had no fever and showed improved neck rigidity. The results of CSF routine and biochemical tests showed significant improvement (Figures 1 and 2), and Staphylococcus epidermidis was isolated from the CSF culture. On May 12, given the isolation of Staphylococcus epidermidis from several CSF cultures and the poor outcome of vancomycin treatment, anti-infective treatment with linezolid was resumed, combined with entecavir ( $0.5 \mathrm{mg}$ once a day P.O.) for antiHBV (hepatitis B virus) treatment. A repeat blood biochemical test on May 16 showed $58 \mathrm{U} / \mathrm{L}$ AST and $125 \mathrm{U} / \mathrm{L}$ ALT. On May 21, the number of platelet count was $182 \times 10^{9} /$ L. On May 24 (d64), routine CSF tests showed normal results (Figure 1). On May 25, repeat head and lung CT scans showed left thalamic post-haemorrhagic encephalomalacia, while the rest were similar to previous results.

On May 26, the patient was in the twilight state of consciousness. After tracheotomy, the patient exhibited smooth spontaneous breathing and was supported by liquid diet. The patient had no fever, and there was a slight improvement of neck rigidity. The antibacterial drugs, including linezolid and ceftazidime, were discontinued. On June 1, the patient regained consciousness and was transferred to the Department of Rehabilitation Medicine for comprehensive rehabilitation. On June 16, the number of platelet count was $269 \times 10^{9} / \mathrm{L}$. On June 28 , the recovery was satisfactory and the patient was discharged from hospital. The treatment regimens and CSF cell counts, proportion of multinucleated cells during hospitalization in Department of Neurosurgery are shown in Figure 1. The trends of concentration of protein and sugar in CSF are shown in Figure 2.

\section{Discussion}

For the treatment of intracranial infections caused by MRSA/MRCoNS, guidelines of ESCMID (European Society for Clinical Microbiology and Infectious Diseases) and the IDSA all recommend vancomycin as a first-line therapeutic agent. ${ }^{2,3}$ Previously, trough concentration is recommended as $\mathrm{PK} / \mathrm{PD}$ parameter for evaluating efficacy of vancomycin, and for complicated infections, trough concentration should be maintained at 15-20 $\mu \mathrm{g} / \mathrm{mL} .^{4}$ The 2020 Chinese Update vancomycin guideline still recommend trough concentration as a target $\mathrm{PK} / \mathrm{PD}$ value to evaluate vancomycin efficacy. ${ }^{5}$ However, the updated 2020 IDSA guideline indicates that the reliability of utilizing trough concentration to evaluate the efficacy of vancomycin could be affected by various PK parameters such as MIC, apparent distribution volume, age, gender, weight, height. ${ }^{6}$ Therefore, the 2020 version of IDSA guideline recommends AUC-guided dosing and monitoring as a basis for optimal management of vancomycin. For serious MRSA infections, it is recommended to target an AUC/MIC ratio of 400 to 600 (assuming a vancomycin MIC of $1 \mathrm{mg} / \mathrm{L}$ ), and trough concentration of $15-20 \mu \mathrm{g} / \mathrm{mL}$ is no longer recommended as a target value alone.

In our case, the vancomycin trough serum concentration was $18.3 \mu \mathrm{g} / \mathrm{mL}$, within the range of $15-20 \mu \mathrm{g} / \mathrm{mL}$, and the vancomycin concentration in CSF was $5.0 \mu \mathrm{g} / \mathrm{mL}$. The plasma AUC was calculated to be $250 \mathrm{~h} \bullet \mu \mathrm{g} / \mathrm{mL}$ using nonlinear mixed-effect model software program (NONMEM, version 7.2), so the AUC/MIC ratio was $125(\mathrm{MIC}=2 \mu \mathrm{g} / \mathrm{mL})$, unable to reach the target AUC/ MIC. There is no relationship between CSF-to-serum ratios of vancomycin and clinical cure in CNS infections. ${ }^{7}$ In our case, the CSF-to-serum ratio of vancomycin is 0.27 , the therapeutic effect was unsatisfactory. Our results further verify that AUC/MIC is a more appropriate $\mathrm{PK} / \mathrm{PD}$ parameter for evaluating the efficacy of vancomycin.

Moreover, if the MIC of vancomycin against MRSA/ MRCoNS is greater than $1 \mu \mathrm{g} / \mathrm{mL}$, vancomycin can be 
replaced with linezolid, daptomycin, or sulfamethoxazole. ${ }^{1}$ Linezolid has been widely and successfully used in patients with CSF shunt infection. ${ }^{8-10}$ The patient in our case was considered to be hospital-acquired intracranial infection due to ventricular drainage. The patient was treated with vancomycin for 36 days although no improvement was observed. Eventually, the patient was cured by switching vancomycin to linezolid. We further searched the available literature reporting linezolid regimen for treating intracranial infections caused by MRSA/ MRCoNS. The clinical characteristics of patients reported in these literature, including vancomycin/ linezolid MIC values of Staphylococcus, were shown in Table 1. Among the 23 cases in Table 1, 14 were caused by MRSA, 8 by MRCoNS, and 1 by VISA. Nineteen patients recovered fully, 3 patients achieved microbiological clearance but ultimately died of comorbidities, and treatment failed in 1 patient. One case had a vancomycin MIC of $1 \mu \mathrm{g} / \mathrm{mL}$ against MRSA/MRCoNS, 22 cases had an MIC $>1 \mu \mathrm{g} /$ $\mathrm{mL}$, so linezolid can achieve a good therapeutic effect when the vancomycin MIC $>1 \mu \mathrm{g} / \mathrm{mL}$.

The antimicrobial physicochemical properties (such as molecular weight, hydrophilicity, and plasma protein binding rate), the pathophysiology of the peripheral disorders of CNS (such as BBB disruption and blood-brain inflammation), the PK/PD of antimicrobials (time-dependence or concentration-dependence), and bacterial susceptibility should all be considered in the selection of antimicrobials for CNS infections. ${ }^{11}$ The molecular weight of linezolid is 337.35 , which is much smaller than vancomycin and teicoplanin, and its plasma protein binding ratio is $31 \%$. After oral intake of $600 \mathrm{mg}$, the steady-state peak serum concentration $\left(\mathrm{C}_{\max }\right)$ of linezolid is $15-27 \mu \mathrm{g} / \mathrm{mL}$ with a high tissue concentration. ${ }^{12}$ The $\mathrm{CSF} /$ plasma AUC ratio of linezolid is 0.5 to 0.9 when there is no inflammation of the meninges, and 0.7 when there is inflammation of the meninges. ${ }^{11}$ The concentration of linezolid in CSF can exceed the MIC against Gram-positive bacteria that cause most intracranial infections. Although linezolid is a bacteriostatic agent, there are several case reports of its use in the management of severe Gram-positive bacterial infection, such as meningitis and endocarditis, where bactericidal activity is traditionally considered to be necessary. ${ }^{13}$

The linezolid MIC of MRSA/MRCoNS was reported in 15 cases, of which one case of MRSA had a linezolid MIC of $4 \mu \mathrm{g} / \mathrm{mL}$. The patient was treated with linezolid combined with daptomycin and rifampicin and was cured.
One case of MRCoNS had a linezolid MIC of $1.5 \mu \mathrm{g} / \mathrm{mL}$, and the patient was cured by linezolid combination therapy with rifampicin. The remaining cases of MRSA/MRCoNS all had linezolid MIC $\leq 1 \mu \mathrm{g} / \mathrm{mL}$, and all patients were cured after treatment with linezolid monotherapy. In our case, the linezolid MIC of MRSE was $4 \mu \mathrm{g} / \mathrm{mL}$, and the patient was eventually cured after treatment with linezolid monotherapy. Linezolid is a time-dependent antibacterial drug, AUC/MIC and $\% \mathrm{~T}_{>\text {MIC }}$ are the PK/PD parameters to determine its efficacy. ${ }^{14}$ A Monte Carlo simulation study of linezolid in plasma/CSF, linezolid was routinely administered at a dose of $0.6 \mathrm{~g}$ every $12 \mathrm{~h} .{ }^{15}$ When $\mathrm{AUC}_{0 \sim 24 \mathrm{~h}}$ /MIC $\geq 59.1$ was applied as a target PK/PD index, the probability of target attainment (PTA) for linezolid in plasma was $\geq 90 \%$ for pathogens only with a MIC of $\leq 2$ $\mu \mathrm{g} / \mathrm{mL}$, whereas the PTA $\geq 90 \%$ in CSF could be reached with a MIC of $\leq 1 \mu \mathrm{g} / \mathrm{mL}^{15}$ When $\% \mathrm{~T}_{>\mathrm{MIC}} \geq 40 \%$ was applied as a target PK/PD index, the PTA of linezolid in plasma/CSF was excellent when the MIC was $\leq 4 \mu \mathrm{g} / \mathrm{mL}^{15}$ In the study reported by Beer et al, when the linezolid MICs were $2 \mu \mathrm{g} / \mathrm{mL}$ and $4 \mu \mathrm{g} / \mathrm{mL}$, the $\% \mathrm{~T}_{>\text {MIC }}$ in CSF were $99.8 \%$ and $57.2 \%$, respectively. ${ }^{16}$ Therefore, the conventional dose of linezolid can achieve good therapeutic effect when the linezolid MIC is $\leq 2 \mu \mathrm{g} / \mathrm{mL}$. When the linezolid MIC is $4 \mu \mathrm{g} / \mathrm{mL}$ or the patient is critically ill, it may be necessary to increase the dose, dosing frequency, or the duration of intravenous drip to improve the therapeutic effect. ${ }^{15}$ Monitoring drug concentration will facilitate the adjustment of dosing to achieve better treatment effect. ${ }^{15}$ In addition, combination therapy may help to control and eradicate pathogens in time. In the study reported by Kelesidis et al, the triple combination of linezolid, daptomycin and rifampicin had synergistic bactericidal effect in vitro. ${ }^{17}$ This regimen may be attempted for salvage treatment. Moreover, the combination of linezolid with rifampicin and the combination of linezolid with vancomycin also revealed synergistic effects in vitro, with case reports on successful clinical applications. ${ }^{18}$ However, combination therapy do not always get favourable outcomes, and further studies are necessary to confirm the efficacy of combination drug regimens.

Hepatitis B surface antigen, hepatitis B e antibody and hepatitis B core antibody were all positive in the patient, with positive HBV DNA $\left(95 \times 10^{3} \mathrm{IU} / \mathrm{mL}\right)$, leading to the diagnosis of HBeAg-negative chronic hepatitis B. Transaminases were elevated in the patient before linezolid therapy. Linezolid therapy was initiated on May 6 and had been only used for 4 days when a progressive 


\begin{tabular}{|c|c|c|c|c|c|c|c|c|c|c|c|c|}
\hline 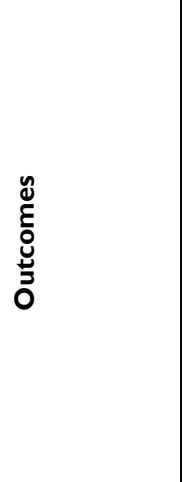 & 怘 & $\begin{array}{l}\stackrel{\square}{0} \\
\stackrel{\Xi}{3}\end{array}$ & 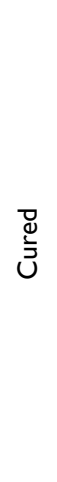 & 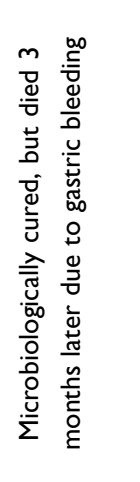 & 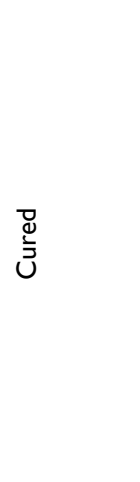 & 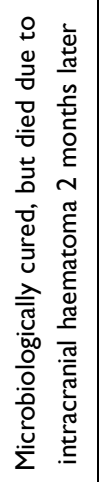 & 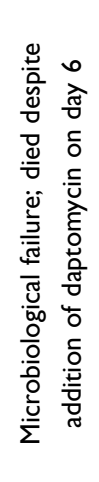 & 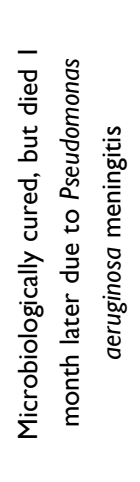 & 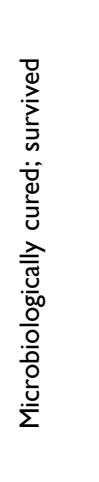 & 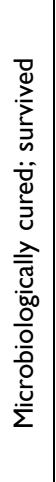 & 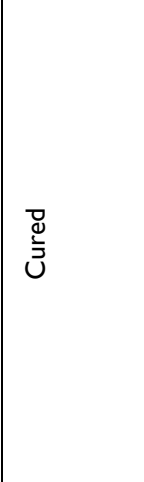 & 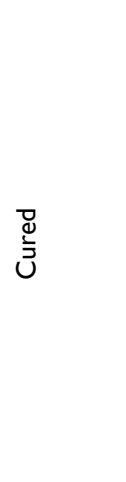 \\
\hline 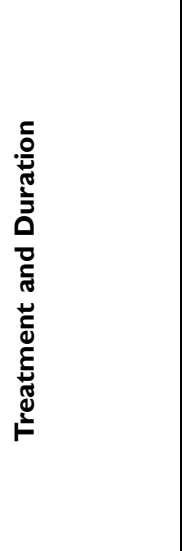 & 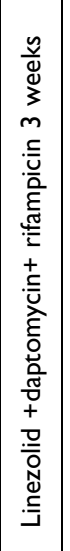 & 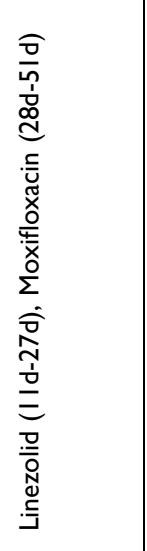 & 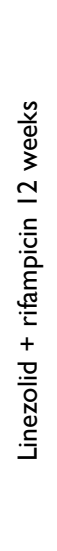 & 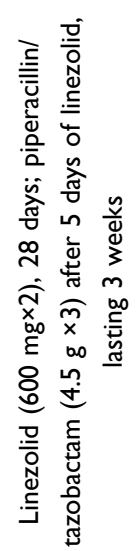 & 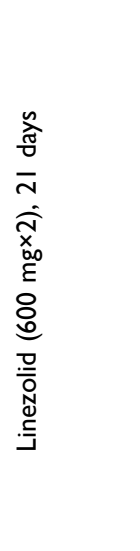 & 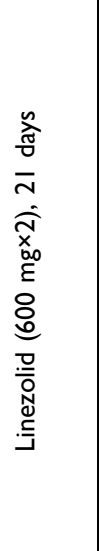 & 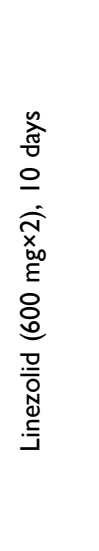 & 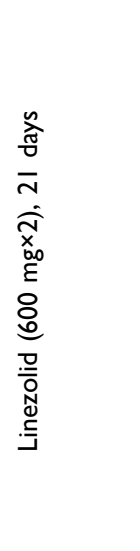 & 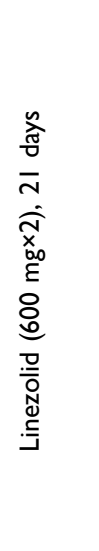 & 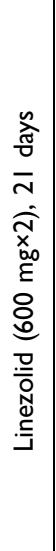 & 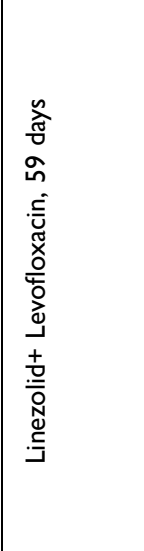 & 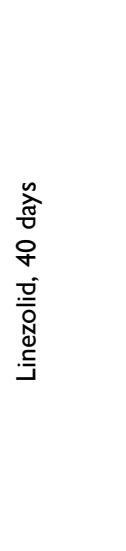 \\
\hline 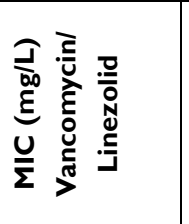 & \pm & $\underset{\checkmark}{\mathbb{Z}}$ & $\stackrel{n ?}{\frac{n}{\gamma}}$ & $\underset{N}{\mathbb{Z}}$ & $\underset{N}{\mathbb{Z}}$ & $\underset{N}{\mathbb{Z}}$ & $\underset{N}{\mathbb{Z}}$ & $\underset{N}{\mathbb{Z}}$ & $\underset{N}{\mathbb{Z}}$ & $\underset{\sim}{\mathbb{Z}}$ & 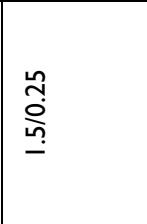 & $\bar{\lambda}$ \\
\hline 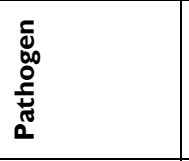 & 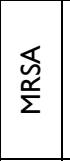 & $\stackrel{\overleftarrow{n}}{>}$ & $\begin{array}{l}n \\
\mathcal{u} \\
0 \\
\frac{u}{\Sigma}\end{array}$ & $\begin{array}{l}\overleftarrow{\alpha} \\
\stackrel{\underline{\alpha}}{\Sigma}\end{array}$ & 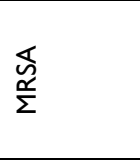 & 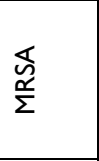 & $\begin{array}{l}\overleftarrow{x} \\
\frac{1}{\Sigma}\end{array}$ & $\begin{array}{l}\overleftarrow{\nwarrow} \\
\stackrel{\Sigma}{\Sigma}\end{array}$ & $\begin{array}{l}\varkappa_{0} \\
\text { Un } \\
\frac{\alpha}{\Sigma}\end{array}$ & $\begin{array}{l}\varkappa_{0} \\
0 \\
\mathcal{u} \\
\Sigma\end{array}$ & $\begin{array}{l}\mathbb{\Lambda} \\
\text { 足 }\end{array}$ & 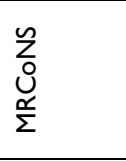 \\
\hline 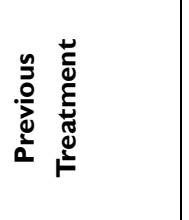 & 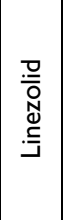 & 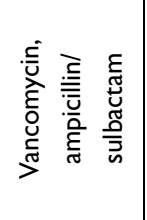 & 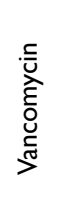 & 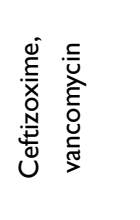 & 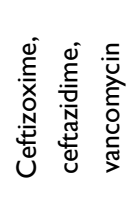 & 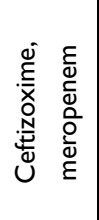 & 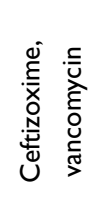 & 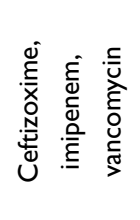 & 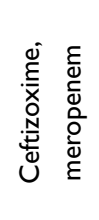 & 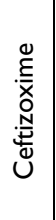 & 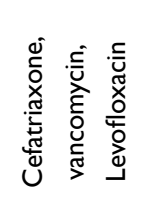 & 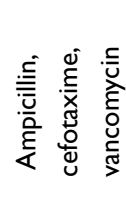 \\
\hline 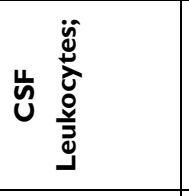 & 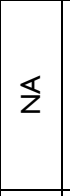 & 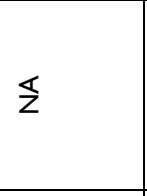 & 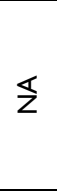 & $\frac{1}{\frac{0}{x}}$ & $\frac{1}{\frac{0}{x}}$ & $\begin{array}{l}\stackrel{1}{a} \\
\frac{0}{x} \\
\stackrel{2}{0} \\
0\end{array}$ & $\begin{array}{l}\frac{1}{0} \\
\frac{0}{x} \\
\frac{x}{1}\end{array}$ & 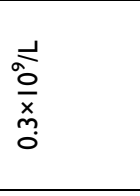 & $\begin{array}{l}1 \\
0 \\
\frac{0}{x} \\
0 \\
0\end{array}$ & $\begin{array}{l}\frac{1}{2} \\
\frac{0}{x} \\
0 \\
m \\
0\end{array}$ & 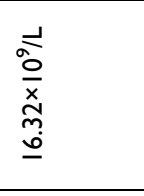 & $\begin{array}{l}\frac{1}{0} \\
\frac{0}{x} \\
\frac{0}{0} \\
0\end{array}$ \\
\hline 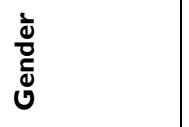 & $\frac{0}{\Sigma}$ & $\frac{0}{\sum}$ & $\begin{array}{l}\frac{0}{\tilde{\Xi}} \\
\frac{\tilde{\Psi}}{\mathscr{W}}\end{array}$ & $\frac{0}{\Sigma}$ & $\begin{array}{l}\frac{\mathscr{\circlearrowright}}{\tilde{\varpi}} \\
\frac{\tilde{\Psi}}{\llcorner}\end{array}$ & $\frac{\frac{0}{\pi}}{\Sigma}$ & $\frac{0}{\Sigma}$ & 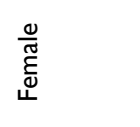 & 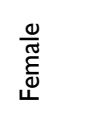 & $\frac{\frac{\omega}{\sigma e}}{\Sigma}$ & $\frac{0}{\Sigma}$ & $\begin{array}{l}\frac{\mathscr{\circlearrowright}}{\tilde{\varpi}} \\
\frac{\tilde{\Psi}}{\llcorner}\end{array}$ \\
\hline$\underset{\&}{8}$ & 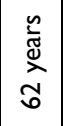 & 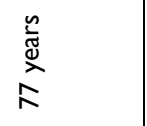 & 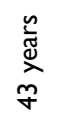 & 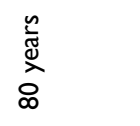 & 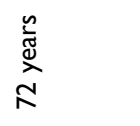 & 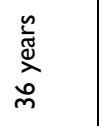 & 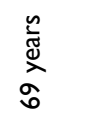 & 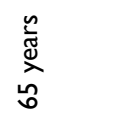 & 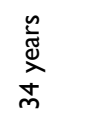 & 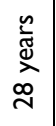 & 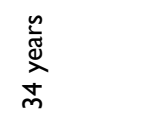 & $\begin{array}{l}\text { 令 } \\
\text { స̃ }\end{array}$ \\
\hline 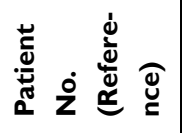 & 틀 & $\underset{\sim}{\stackrel{\Xi}{\sim}}$ & 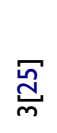 & $\frac{\bar{m}}{\vec{\psi}}$ & $\stackrel{\bar{m}}{\underline{n}}$ & 흥 & $\stackrel{\underline{p}}{\stackrel{p}{k}}$ & $\stackrel{\bar{m}}{\stackrel{\infty}{\delta}}$ & $\frac{\bar{m}}{a}$ & $\underline{\underline{m}}$ & $\stackrel{\vec{D}}{=}$ & 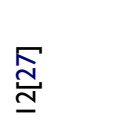 \\
\hline
\end{tabular}




\begin{tabular}{|c|c|c|c|c|c|c|c|c|c|c|}
\hline 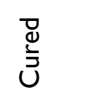 & 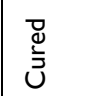 & 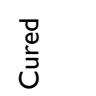 & 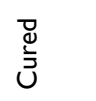 & 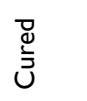 & 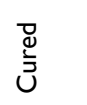 & 总 & 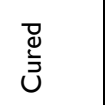 & 怤 & 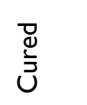 & 导 \\
\hline 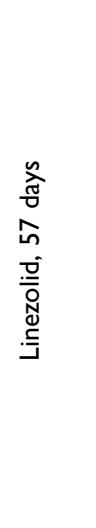 & 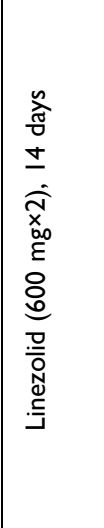 & 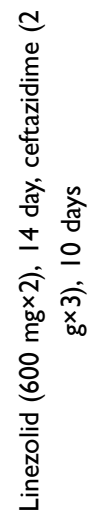 & 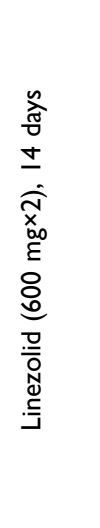 & 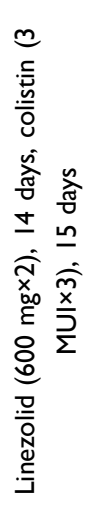 & 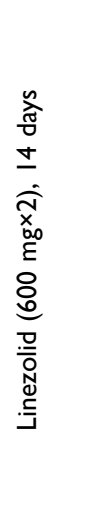 & 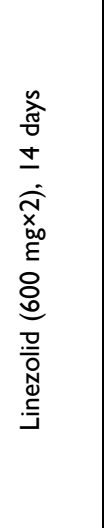 & 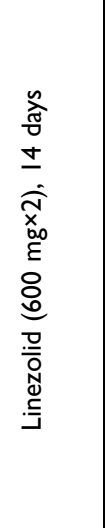 & 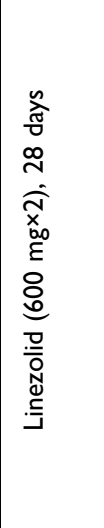 & 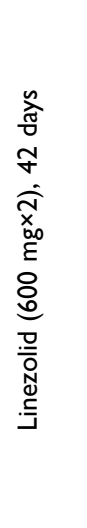 & 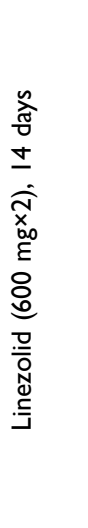 \\
\hline $\bar{\lambda}$ & $\bar{\lambda}$ & $\bar{\lambda}$ & $\bar{\lambda}$ & $\bar{\lambda}$ & $\bar{\lambda}$ & $\bar{\lambda}$ & $\bar{\lambda}$ & $\bar{\lambda}$ & $\bar{\lambda}$ & $\bar{\lambda}$ \\
\hline 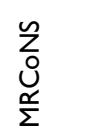 & 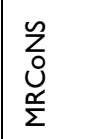 & $\begin{array}{l}z_{0}^{n} \\
\mathcal{O} \\
\frac{\tilde{L}}{\Sigma}\end{array}$ & $\begin{array}{l}\mathbb{\widetilde { \alpha }} \\
\frac{\omega}{\Sigma} \\
\frac{\omega}{\Sigma}\end{array}$ & $\begin{array}{l}\frac{\mathbb{s}}{\alpha} \\
\frac{\alpha}{\Sigma}\end{array}$ & $\begin{array}{l}\stackrel{\Phi}{\alpha} \\
\frac{\alpha}{\Sigma}\end{array}$ & $\begin{array}{l}\sum_{0} \\
\mathcal{O} \\
\frac{\aleph}{\Sigma}\end{array}$ & 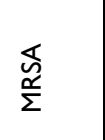 & $\begin{array}{l}\overleftarrow{\overleftarrow{s}} \\
\frac{\alpha}{\Sigma}\end{array}$ & 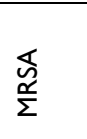 & $\begin{array}{l}\text { 崩 } \\
\frac{\tilde{x}}{\Sigma}\end{array}$ \\
\hline 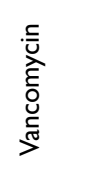 & 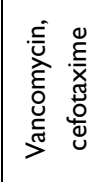 & 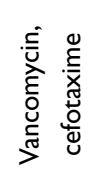 & 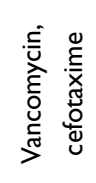 & 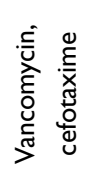 & 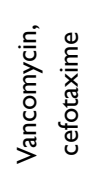 & 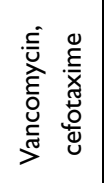 & 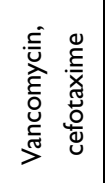 & 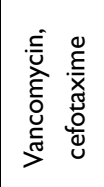 & 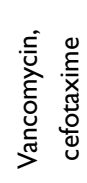 & 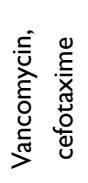 \\
\hline$\frac{\frac{1}{0}}{\frac{0}{x}}$ & 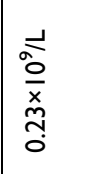 & $\begin{array}{l}\stackrel{1}{\circ} \\
\frac{0}{x} \\
\stackrel{\circ}{\circ} \\
\frac{h}{0}\end{array}$ & 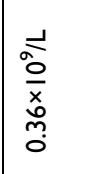 & & $\begin{array}{l}\frac{5}{0} \\
\frac{0}{x} \\
\frac{0}{5}\end{array}$ & $\begin{array}{l}\frac{1}{0} \\
\frac{0}{x} \\
\frac{\infty}{0} \\
\frac{0}{0}\end{array}$ & 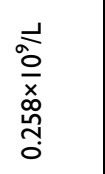 & $\mathbb{z}$ & $\underline{z}$ & $\begin{array}{l}\frac{\downarrow}{\infty} \\
\frac{0}{x} \\
\infty\end{array}$ \\
\hline 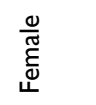 & 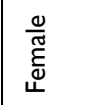 & $\frac{\frac{\rho}{\tilde{\omega}}}{\Sigma}$ & $\frac{0}{\Sigma}$ & 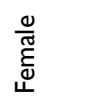 & $\frac{\frac{0}{\pi}}{\Sigma}$ & 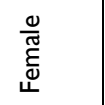 & 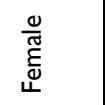 & $\frac{\frac{0}{\Sigma}}{\Sigma}$ & $\frac{\frac{\varrho}{\sigma}}{\Sigma}$ & 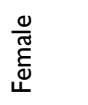 \\
\hline$=\frac{\stackrel{n}{\underline{t}}}{\underline{\underline{t}}}$ & 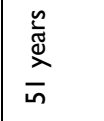 & 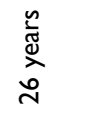 & 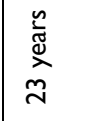 & 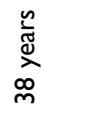 & 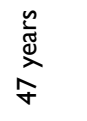 & 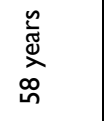 & 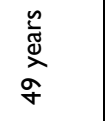 & 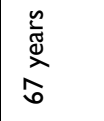 & 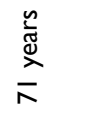 & 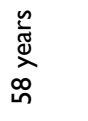 \\
\hline$\underset{\underline{\underline{D}}}{\stackrel{\Sigma}{d}}$ & 离 & 离 & 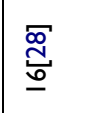 & $\stackrel{\mathbb{D}}{\underline{\Sigma}}$ & $\begin{array}{l}\underset{\mathbb{D}}{\tilde{D}} \\
\underline{\underline{m}}\end{array}$ & 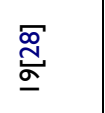 & 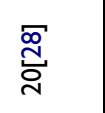 & $\stackrel{\underset{d}{\sim}}{\stackrel{D}{N}}$ & 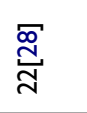 & 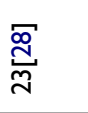 \\
\hline
\end{tabular}


increase in transaminases was observed. The possibility of linezolid-induced liver injury seem not to be ruled out. However, previous studies demonstrated that drug-induced liver injury (DILI) with fulminant liver failure and lactic acidosis were probably related to prolonged linezolid exposure. ${ }^{19}$ In our case, the Roussel Uclaf Causality Assessment Method (RUCAM) yielded a score of 0 . Moreover, the liver function gradually improved with subsequent treatment with linezolid and hepatoprotective. Therefore, the liver injury in our case was not considered to be associated with linezolid.

In our case, linezolid was administrated in normal dose. No significant adverse reactions were detected, and the patient was eventually cured. From this report, the dosage of linezolid do not appear to be related to clinical cure of CNS infections, and if the subsequent treatment is satisfactory, the initial normal dose does not necessarily need to be increased.

The common side effects of linezolid include thrombocytopenia, anemia, neutropenia, gastrointestinal disorders, liver dysfunction, lactic acidosis. ${ }^{20}$ Specially, linezolid-induced thrombocytopenia can increase risk of gastrointestinal and intracranial haemorrhage. ${ }^{21,22}$ In our report, no significant decrease in platelet count could be observed during the entire course of linezolid therapy at normal dosage. Besides, no serious adverse reactions occurred during linezolid therapy in any of the 23 patients reported in literature. However, the patients with intracranial infection often require linezolid prolonged administration, high attention also need be paid to the adverse reactions of linezolid. Close platelet count monitoring is required for the patients who receive linezolid therapy longer than a week, and have impaired renal function or low baseline platelet counts. ${ }^{23}$

\section{Conclusion}

Vancomycin dosing should be based on AUC-guided dosing and monitoring. The best predictor of vancomycin efficacy is AUC/MIC, and trough concentration should not be used as a substitute. For patients with serious MRSA/MRCoNS infections, it is recommended to target an AUC/MIC ratio of 400 to 600 to achieve clinical efficacy. When the vancomycin MIC of MRSA/MRCoNS is greater than $1 \mu \mathrm{g} / \mathrm{mL}$, the target AUC/MIC may not be achieved. In such cases, linezolid can be used to obtain good therapeutic effect.

\section{Abbreviations}

PK/PD, pharmacokinetic/pharmacodynamic; AUC, area under the curve; MIC, Minimum Inhibitory Concentration;
MRSE, methicillin-resistant Staphylococcus epidermidis; MRSA, methicillin-resistant Staphylococcus aureus; MRCoNS, methicillin-resistant coagulase-negative Staphylococcus; BBB, blood-brain barrier; CNS, central nervous system; IDSA, Infectious Diseases Society of America; HCAMV, health care-associated meningitis or ventriculitis; WBC, white blood cell; CRP, C-reactive protein; CSF, cerebrospinal fluid; BMD, broth microdilution; PTA, probability of target attainment; ALT, alanine transaminase; AST, aspartate transaminase; PTA, probability of target attainment; DILI, drug-induced liver injury.

\section{Data Sharing Statement}

The data used and/or analyzed during the current study are available from the corresponding author upon a reasonable request.

\section{Ethics Approval and Consent to Participate}

This study was approved by the ethical committee of Quanzhou First Hospital Affiliated to Fujian Medical University (Ethics number: QZFH.FJMU.2020.169).

\section{Patient Consent for Publication}

Written informed consent was obtained from the patient and the patient's close relatives for publication of this Case report and accompanying images, without identifying information.

\section{Acknowledgments}

The authors gratefully acknowledgement all the medical staff in the Department of Neurosurgery of Quanzhou First Hospital Affiliated to Fujian Medical University, for the collection of clinical specimens.

\section{Author Contributions}

All authors made a significant contribution to the work reported, whether that is in the conception, study design, execution, acquisition of data, analysis and interpretation, or in all these areas; took part in drafting, revising or critically reviewing the article; gave final approval of the version to be published; have agreed on the journal to which the article has been submitted; and agree to be accountable for all aspects of the work.

\section{Funding}

This work was supported by Natural Science Foundation of Fujian province (2019J01593), High-level Talent 
Innovation Project of Quanzhou (2018C067R), Science and Technology Innovation Joint Project of Fujian province (2019Y9048), and Science and Technology Project of Quanzhou (2018Z045, 2018Z069, 2018Z074).

\section{Disclosure}

The authors declare that they have no competing interests.

\section{References}

1. van de Beek D, Drake JM, Tunkel AR. Nosocomial bacterial meningitis. $N$ Engl J Med. 2010;362(2):146-154. doi:10.1056/ NEJMra0804573

2. Tunkel AR, Hasbun R, Bhimraj A, et al. 2017 Infectious diseases society of america's clinical practice guidelines for healthcareassociated ventriculitis and meningitis. Clin Infect Dis. 2017;64(6): e34-e65. doi:10.1093/cid/ciw861

3. van de Beek D, Cabellos C, Dzupova O, et al. ESCMID guideline: diagnosis and treatment of acute bacterial meningitis. Clin Microbiol Infect. 2016;22(Suppl 3):S37-62. doi:10.1016/j.cmi.2016.01.007

4. Rybak M, Lomaestro B, Rotschafer JC, et al. Therapeutic monitoring of vancomycin in adult patients: a consensus review of the American Society of health-system pharmacists, the infectious diseases society of America, and the society of infectious diseases pharmacists. $\mathrm{Am}$ $J$ Health Syst Pharm. 2009;66(1):82-98. doi:10.2146/ajhp080434

5. He N, Su S, Ye Z, et al. Evidence-based guideline for therapeutic drug monitoring of vancomycin: 2020 update by the division of therapeutic drug monitoring, Chinese pharmacological society. Clin Infect Dis. 2020;71(Suppl 4):S363-S371. doi:10.1093/cid/ciaa1536

6. Rybak MJ, Le J, Lodise TP, et al. Therapeutic monitoring of vancomycin for serious methicillin-resistant Staphylococcus aureus infections: a revised consensus guideline and review by the American society of health-system pharmacists, the infectious diseases society of America, the pediatric infectious diseases society, and the society of infectious diseases pharmacists. Am J Health Syst Pharm. 2020;77 (11):835-864. doi:10.1093/ajhp/zxaa036

7. Beach JE, Perrott J, Turgeon RD, et al. Penetration of vancomycin into the cerebrospinal fluid: a systematic review. Clin Pharmacokinet. 2017;56(12):1479-1490. doi:10.1007/s40262-017-0548-y

8. Yilmaz A, Dalgic N, Müslüman M, et al. Linezolid treatment of shunt-related cerebrospinal fluid infections in children. $J$ Neurosurg Pediatr. 2010;5(5):443-448. doi:10.3171/2009.12.PEDS09421

9. Maranich AM, Rajnik M. Successful treatment of vancomycin-resistant enterococcal ventriculitis in a pediatric patient with linezolid. Mil Med. 2008;173(9):927-929. doi:10.7205/ milmed.173.9.927

10. Graham PL, Ampofo K, Saiman L. Linezolid treatment of vancomycin-resistant Enterococcus faecium ventriculitis. Pediatr Infect Dis J. 2002;21(8):798-800. doi:10.1097/00006454200208000-00025

11. Di Paolo A, Gori G, Tascini C, et al. Clinical pharmacokinetics of antibacterials in cerebrospinal fluid. Clin Pharmacokinet. 2013;52 (7):511-542. doi:10.1007/s40262-013-0062-9

12. Dryden MS. Linezolid pharmacokinetics and pharmacodynamics in clinical treatment. J Antimicrob Chemother. 2011;66(Suppl 4):iv7iv15. doi:10.1093/jac/dkr072

13. Sipahi OR, Bardak S, Turhan T, et al. Linezolid in the treatment of methicillin-resistant staphylococcal post-neurosurgical meningitis: a series of 17 cases. Scand J Infect Dis. 2011;43(10):757-764. doi:10.3109/00365548.2011.585177
14. Andes D, van Ogtrop ML, Peng J, et al. In vivo pharmacodynamics of a new oxazolidinone (linezolid). Antimicrob Agents Chemother. 2002;46(11):3484-3489. doi:10.1128/aac.46.11.3484-3489.2002

15. Wu X, Tang Y, Zhang X, et al. Pharmacokinetics and pharmacodynamics of linezolid in plasma/cerebrospinal fluid in patients with cerebral hemorrhage after lateral ventricular drainage by Monte Carlo simulation. Drug Des Devel Ther. 2018;12:1679-1684. doi:10.2147/DDDT.S168757

16. Beer R, Engelhardt KW, Pfausler B, et al. Pharmacokinetics of intravenous linezolid in cerebrospinal fluid and plasma in neurointensive care patients with staphylococcal ventriculitis associated with external ventricular drains. Antimicrob Agents Chemother. 2007;51 (1):379-382. doi:10.1128/AAC.00515-06

17. Kelesidis T, Humphries R, Ward K, et al. Combination therapy with daptomycin, linezolid, and rifampin as treatment option for MRSA meningitis and bacteremia. Diagn Microbiol Infect Dis. 2011;71 (3):286-290. doi:10.1016/j.diagmicrobio.2011.07.001

18. Malacarne P, Viaggi B, Dip A, et al. Linezolid cerebrospinal fluid concentration in central nervous system infection. $J$ Chemother. 2007;19(1):90-93. doi:10.1179/joc.2007.19.1.90

19. De Bus L, Depuydt P, Libbrecht L, et al. Severe drug-induced liver injury associated with prolonged use of linezolid. J Med Toxicol. 2010;6(3):322-326. doi:10.1007/s13181-010-0047-0

20. Auriti C, Piersigilli F, Bersani I, et al. Staphylococcal meningitis therapy with linezolid in a young infant: efficacy, CSF levels and side effects. Ital J Pediatr. 2020;46(1):90. doi:10.1186/s13052-02000854-z

21. Kim HS, Kim H, Jeong YJ, et al. Comparative analysis of the suspected heparin-induced thrombocytopenia level in Korea. Basic Clin Pharmacol Toxicol. 2017;121(4):360-367. doi:10.1111/ bcpt.12791

22. Vandijck DM, Blot SI, De Waele JJ, et al. Thrombocytopenia and outcome in critically ill patients with bloodstream infection. Heart Lung. 2010;39(1):21-26. doi:10.1016/j.hrtlng.2009.07.005

23. Choi GW, Lee JY, Chang MJ, et al. Risk factors for linezolid-induced thrombocytopenia in patients without haemato-oncologic diseases. Basic Clin Pharmacol Toxicol. 2019;124(2):228-234. doi:10.1111/ bcpt. 13123

24. Kino H, Suzuki H, Yamaguchi T, et al. Central nervous system infection caused by vancomycin-intermediate Staphylococcus aureus (SCCmec type IV, ST8). J Infect Chemother. 2014;20(10):643-646. doi:10.1016/j.jiac.2014.06.008

25. Kruse AJ, Peerdeman SM, Bet PM, et al. Successful treatment with linezolid and rifampicin of meningitis due to methicillin-resistant Staphylococcus epidermidis refractory to vancomycin treatment. Eur J Clin Microbiol Infect Dis. 2006;25(2):135-137. doi:10.1007/ s10096-006-0097-z

26. Fernández-Ruiz M, Cervera $\mathrm{C}$, Pitart $\mathrm{C}$, et al. Community-acquired methicillin-resistant Staphylococcus aureus meningitis complicated by cerebral infarction. Role of antibiotic combination of linezolid plus levofloxacin. Intern Med. 2010;49(18):1971-1974. doi:10.2169/ internalmedicine.49.3942

27. Ochi F, Tauchi H, Nagai K, et al. Therapeutic effect of linezolid in children with health care-associated meningitis or ventriculitis. Clin Pediatr (Phila). 2018;57(14):1672-1676. doi:10.1177/ 0009922818803399

28. Rebai L, Fitouhi N, Daghmouri MA, et al. Linezolid for the treatment of postneurosurgical infection caused by methicillin-resistant Staphylococcus. Surg Neurol Int. 2019;10:215. doi:10.25259/ SNI_455_2019 


\section{Publish your work in this journal}

Infection and Drug Resistance is an international, peer-reviewed openaccess journal that focuses on the optimal treatment of infection (bacterial, fungal and viral) and the development and institution of preventive strategies to minimize the development and spread of resistance. The journal is specifically concerned with the epidemiology of antibiotic resistance and the mechanisms of resistance development and diffusion in both hospitals and the community. The manuscript management system is completely online and includes a very quick and fair peerreview system, which is all easy to use. Visit http://www.dovepress.com testimonials.php to read real quotes from published authors. 